\title{
Self-Q-Switched Cr:LiCAF laser near 800 nm
}

\author{
Ersen Beyatli ${ }^{\mathrm{a}}$, Alphan Sennaroglu ${ }^{\mathrm{a}}$, and Umit Demirbas ${ }^{* \mathrm{~b}}$ \\ ${ }^{a}$ Laser Research Laboratory, Departments of Physics and Electrical-Electronics Engineering, \\ Koç University, Rumelifeneri, Sariyer, 34450, Istanbul, Turkey \\ ${ }^{\mathrm{b}}$ Laser Technology Laboratory, Department of Electrical and Electronics Engineering, \\ Antalya International University, Dosemealti, Antalya 07190, Turkey
}

\begin{abstract}
Self-Q-switching (SQS) of lasers enable the generation of Q-switched pulses from simple laser cavities without using any additional saturable absorbers or active modulators. Earlier studies have reported SQS in ruby, Nd:YAG, and Cr:LiSAF lasers. However, these systems were mostly flashlamp pumped and required cooling of the crystal and/or misalignment of the laser cavity for the observation of SQS. In this presentation, for the first time to our knowledge, we report SQS operation of a Cr:LiCAF laser. SQS was achieved in an astigmatically compensated x-cavity containing only a Cr:LiCAF crystal that was end-pumped with a 130-mW continuous-wave (cw) diode at $660 \mathrm{~nm}$. During regular $\mathrm{cw}$ operation, the laser produced a diffraction-limited beam with $50 \mathrm{~mW}$ of output power and had a spectral width of $0.5 \mathrm{~nm}$ near $795 \mathrm{~nm}$. SQS operation of the Cr:LiCAF laser could be initiated by fine adjustment of the separation between the curved mirrors of the cavity and was observed at several mirror separations within the stability range of the resonator. During SQS operation, the laser generated pulses with about 5 microsecond duration in the 780-800 nm wavelength range, at repetition rates between 10 and $30 \mathrm{kHz}$. SQS operation was further accompanied with a decrease in the output power to the $30-45 \mathrm{~mW}$ range. In this regime, the output beam became multimode and spectral broadening up to $12.5 \mathrm{~nm}$ (FWHM) was observed.
\end{abstract}

Keywords: Solid-state lasers, Self-Q-Switching of lasers, diode-pumped solid-state lasers, pulsed lasers

\section{INTRODUCTION}

Short laser pulses in the nanosecond to microsecond range are useful for many applications such as material processing, range finding, remote sensing, and nonlinear frequency conversion. Q-switching, which involves the modulation of intracavity losses (or the cavity Q-factor), is one of the widely used techniques for obtaining such short pulses. Intracavity losses can be modulated via active and passive schemes. In active modulation, acousto-optic modulators, electro-optic modulators, or rotating mirrors are typically used to alter the cavity Q-factor. In contrast, optical elements with inherent saturable absorption can also be utilized in passive modulation and do not require any active control. Such saturable absorbers may be based on dyes, semiconductors (AlGaAs, InGaAs, etc..) or transition metal doped materials such as $\mathrm{Cr}^{4+}: \mathrm{YAG}[1]$ and $\mathrm{Cr}^{2+}: \mathrm{ZnSe}[2,3]$.

In1968, I. Freund showed that certain laser cavities can also produce Q-switched behavior without any passive or active modulation elements [4]. This process is now known as self-Q-switching (SQS). A laser cavity operated in the SQS regime simply consists of an optical resonator, a suitable gain medium, and a pump source and generates short pulses under some special conditions. For example, in the initial studies, SQS was observed in flashlamp-pumped ruby lasers [4-7], and to initiate the SQS effect, either the laser cavity mirrors were misaligned [4, 5], or the laser crystal was operated at liquid-nitrogen-temperatures [6,7]. These initial studies employed pulsed pumping[4-7] and Q-switching effect switched the laser output from random spiking to a giant single high-energy laser pulse. Later, SQS was reported in Nd:YAG laser media at $77{ }^{\circ} \mathrm{K}$ [8], in $\mathrm{Nd}: \mathrm{SeOCl}_{2}$ liquid lasers under low feedback conditions (using high output coupling) [9], and in a quasi $\mathrm{cw}$ pumped room-temperature $\mathrm{Nd}: \mathrm{YVO}_{4}$ laser using cavity misalignment [10]. In 1978, A. Szabo demonstrated SQS operation in a cw argon ion laser pumped ruby laser operated at $80{ }^{\circ} \mathrm{K}$ (first demonstration of SQS operation with a $\mathrm{cw}$ pumped system [11]). Since then, SQS operation has been reported in flashlamp pumped Cr:LiSAF lasers [12, 13], Nd: $\mathrm{YVO}_{4}$ microchip lasers [14], Erbium fiber lasers [15], broad area semiconductor lasers

umit79@alum.mit.edu

Solid State Lasers XXII: Technology and Devices, edited by W. Andrew Clarkson, Ramesh K. Shori, Proc. of SPIE Vol. 8599, 85990S - (c) 2013 SPIE · CCC code: 0277-786X/13/\$18 · doi: 10.1117/12.2002964 
[16], Nd:YVO $\mathrm{YHTP}_{4}$ lasers [10], ytterbium fiber lasers [17], and thulium-fiber lasers [18]. Although a large number of studies have been carried out, the underlying mechanism of the SQS effect is not yet fully understood. As an example, for the $\mathrm{Cr}^{+3}$-doped gain media such as ruby and $\mathrm{Cr}$ :LiSAF, earlier studies investigated a nonlinear loss mechanism created by a time-dependent lens occurring inside the gain medium (originating from refractive index changes induced by the population inversion) as a possible reason for the SQS effect [12,13,19-22]. However, a recent study by Godin $e t$ al. reported that the origin of the refractive index change in $\mathrm{Cr}^{+3}$-doped gain media (as well as the underlying physics behind the SQS effect) is not yet clear and requires further investigation [23].

In this study, we report on the SQS operation in Cr:LiCAF gain media for the first time to our knowledge. In our experiments, we used a 4-mirror astigmatically compensated x-cavity. The laser was pumped with a continuouswave (cw) single-spatial-mode diode at $660 \mathrm{~nm}$. As stated earlier, no extra modulation elements was inserted in the cavity. In the regular $\mathrm{cw}$ regime, with an incident pump power of $130 \mathrm{~mW}$, we obtained in excess of $50 \mathrm{~mW}$ of average output power. The laser spectrum was centered around $795 \mathrm{~nm}$ and had a spectral width (full width at half maximum, FWHM) of $0.5 \mathrm{~nm}$. Furthermore, the laser transverse mode was pure $\mathrm{TEM}_{00}$ during $\mathrm{cw}$ operation. However, by fine adjustment of the separation of the cavity curved mirrors, the SQS effect was initiated and was accompanied with a decrease in the output power to $30-45 \mathrm{~mW}$. In this regime, pulses with around 5 microsecond duration were generated with repetition rates of 10-30 kHz. During SQS, a spectral widening also occurred up to $12.5 \mathrm{~nm}$ and the laser transverse beam profile became a mixture of higher-order spatial modes. When we inserted a slit inside the cavity and forced the laser to operate in a single transverse mode, SQS disappeared and the laser switched back to the normal cw operation. As mentioned above, similar effects were observed in different gain media. However, in those systems, pulsed flashlamp pumping was mostly employed and initiating SQS required misalignment of the laser cavity, cooling of the laser crystal to liquid nitrogen temperatures or use of high output coupling. In this study, SQS could be achieved at room temperature, from a low-power $\mathrm{cw}$ diode pumped system and only the curved mirror separation was adjusted to initiate the SQS effect. Moreover, we will also present detailed experimental data on the effect of cavity geometry and pump power on the SQS phenomena and parameters such as SQS repetition frequency, pulse width, and optical spectrum width. We believe that the experimental data presented here will help in better understanding of the underlying physical mechanisms behind the self pulsing phenomena.

\section{EXPERIMENTAL SETUP}

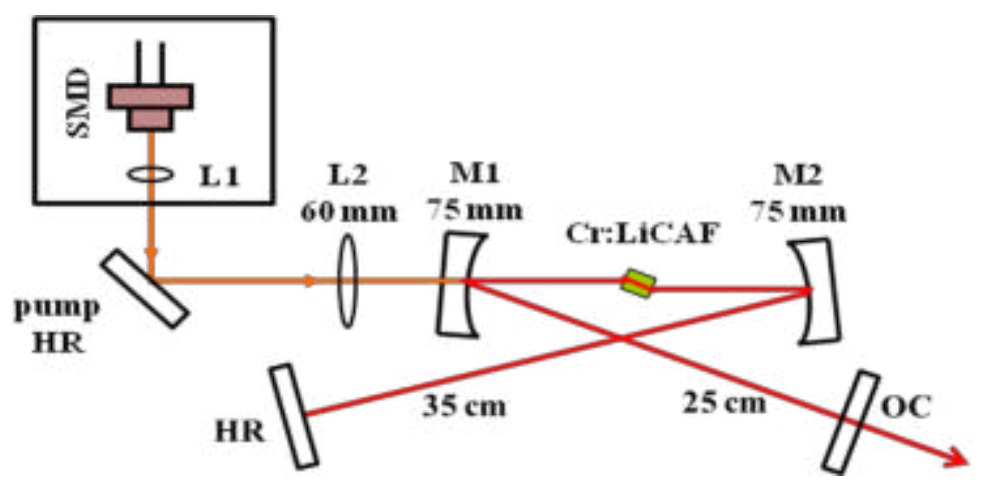

Figure 1 Schematic of the Cr:LiCAF laser pumped with one single-spatial-mode diode (SMD) laser. The laser operated in the SQS regime at specific, discrete values of the separation between the curved mirrors M1 and M2.

In order to attain SQS operation, we used an astigmatically compensated 4-mirror x-cavity with a tight focusing geometry as shown in Fig. 1 [24]. A linearly polarized, single-spatial mode diode pump laser (SMD, Hitachi), capable of producing up to $130 \mathrm{~mW}$ at the driving current of $240 \mathrm{~mA}$, was used to end pump the Cr:LiCAF crystal through the input curved mirror (M1). The output wavelength of the diode laser was around $660 \mathrm{~nm}$. A cylindrical micro lens (L1) was used to obtain a circular beam profile from the diode laser. The pump beam was further collimated with an antireflection (AR) coated aspheric lens that had a focal length of $4.5 \mathrm{~mm}$. An AR-coated input lens (L2) with a focal length of $6 \mathrm{~cm}$ was used to focus the pump beam inside the gain crystal. The laser resonator consisted of two identical intracavity input curved mirrors (M1,M2, with $\mathrm{R}=75 \mathrm{~mm}$ ), a highly reflecting end mirror (HR), and a flat output coupler (OC). The lengths of the high reflector and output coupler arms were set to $35 \mathrm{~cm}$ and $25 \mathrm{~cm}$, respectively, which resulted in a 15-20 $\mu \mathrm{m}$ laser spot inside the crystal. The gain medium, which was a 4-mm-long, uncoated Cr:LiCAF 
crystal with $5 \% \mathrm{Cr}^{3+}$, was positioned at Brewster incidence between M1 and M2. Hence, the Fresnel reflection loss of the pump beam was negligible. With this configuration, the crystal absorbed about $95 \%$ of the pump beam. Neither the pump diode nor the Cr:LiCAF laser crystal required active cooling. Temporal characterization of the laser was performed using a $1 \mathrm{GHz} \mathrm{Si}$ photodetector and a $500 \mathrm{MHz}$ digital sampling oscilloscope. The laser spectrum was measured with a scanning, fiber coupled spectrometer (Ocean Optics, USB2000) and the laser transverse mode was monitored with a beam profiler (Coherent Lasercam HR).

\section{EXPERIMENTAL RESULTS}

The SQS operation was observed at several positions of the cavity stability range by varying the distance between the highly reflecting curved mirrors M1 and M2 (see Fig.1). In order to explore the characteristics of the SQS operation, the output power was measured as a function of the curved mirror separation as shown in Fig. 2. The data were taken at the incident pump power of $130 \mathrm{~mW}$. The cavity output coupler had a transmission of $0.75 \%$. Before taking the data, the pump focusing lens (L2) and the resonator alignment were first optimized at the center of the stability range. Then; the output power was measured by varying only the curved mirror separation.

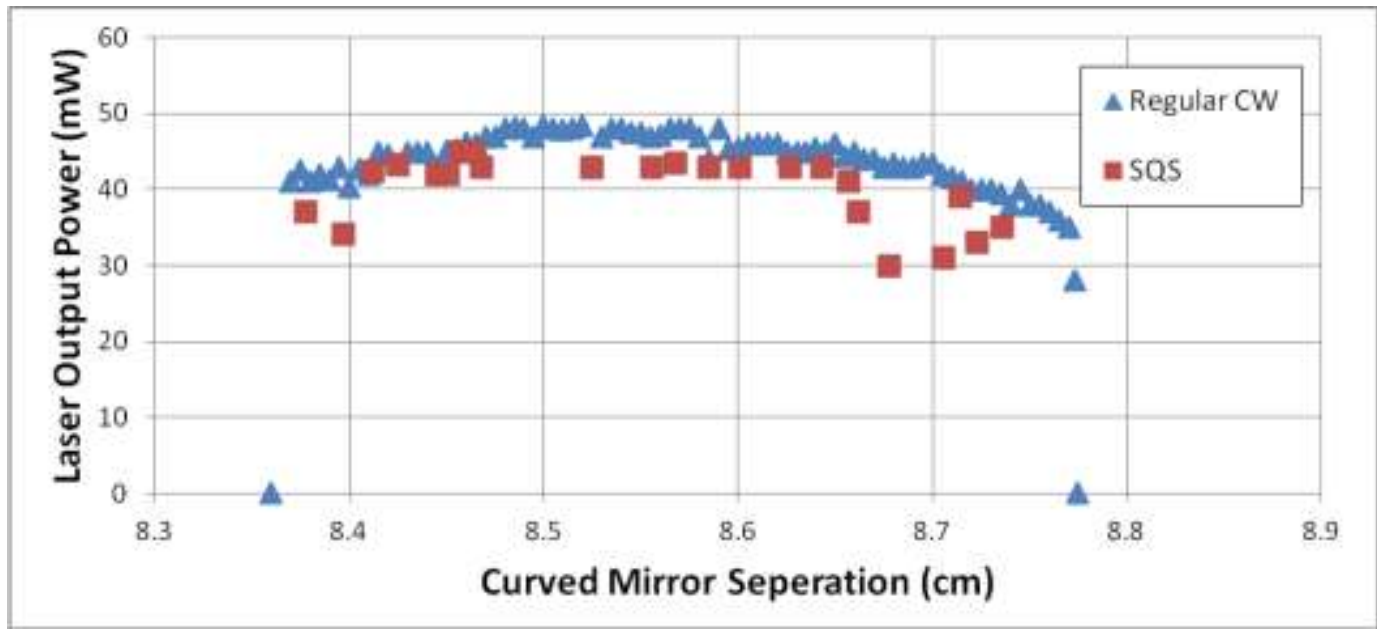

Figure 2 Experimentally measured variation of the laser output power as a function of the curved mirror separation within the outer stability range. (triangles: pure cw operation, squares: SQS operation).

As it can be seen from Fig.2, at most points of the stability region, the laser operated in the continuous-wave $(\mathrm{CW})$ regime (solid triangles in Fig. 2). At these locations, the laser was stable with a $\mathrm{TEM}_{00}$ output and the measured $\mathrm{M}^{2}$ parameter was below 1.05. Average output powers of around $50 \mathrm{~mW}$ could be obtained. Furthermore, in this regime, the spectral widths (FWHM) were about $0.5 \mathrm{~nm}$ near the center wavelength of $795 \mathrm{~nm}$ (see Fig. 3). However, at several positions in the stability range (solid squares in Fig. 2), the laser switched to the SQS regime and continued to operate in that regime in a very stable manner. At these specific positions, the laser output power was in general lower than that during the $\mathrm{cw}$ operation. Moreover, during SQS operation, the laser transverse mode also switched from being singlemode to a structured multimode, and spectral widening was also observed. However, during SQS operation, when we inserted a mechanical slit inside the laser resonator to force the laser to operate in pure $\mathrm{TEM}_{00}$ mode, the laser switched back to pure $\mathrm{cw}$ operation. This was accompanied with an increase in the output power and a decrease in the spectral bandwidth. In the remainder of this section, the rich behavior of the SQS operation at different curved mirror separations will be described in detail.

Figure 4 shows the output characteristics at the curved mirror separation of $8.46 \mathrm{~cm}$ where SQS was observed with $0.75 \%$ output coupling. In this configuration, the output power of the laser decreased from $50 \mathrm{~mW}$ to $43 \mathrm{~mW}$. The laser generated Q-switched pulses with a duration (FWHM) of 4.2 microseconds..The frequency of modulation was $24.4 \mathrm{kHz}$ as can be seen from the fast Fourier transform of the output in Fig. 4(b). The SQS operation also changed the laser output mode from $\mathrm{TEM}_{00}$ to a structured mode (inset figure of 4(b)). The spectral width (FWHM) of the laser spectrum increased from $0.5 \mathrm{~nm}$ to $10 \mathrm{~nm}$ as can be seen from Fig.4(c). The corresponding coherence length at these wavelengths (i.e. $795 \mathrm{~nm}$ ) is about 35 micrometers in air, suggesting that the source could find applications in high axial resolution optical coherence tomography [25]. 


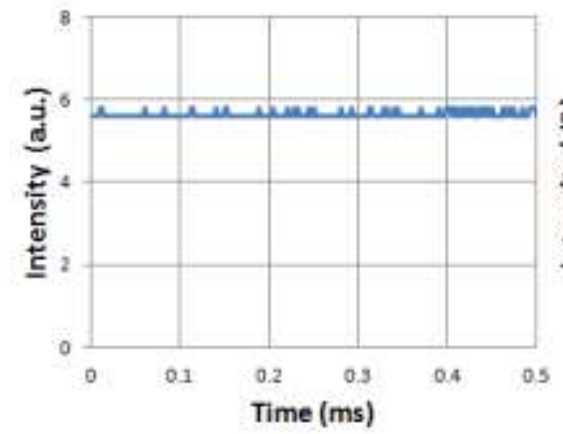

(a)

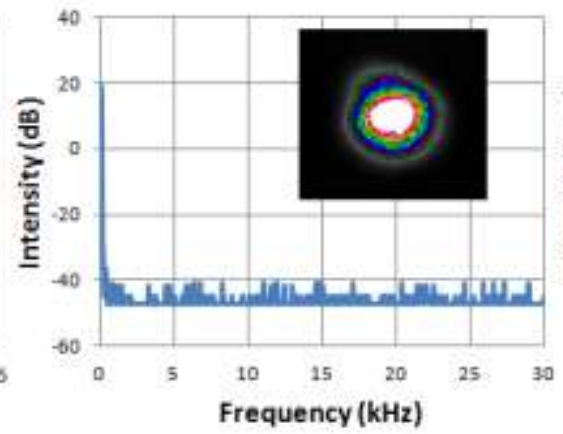

(b)

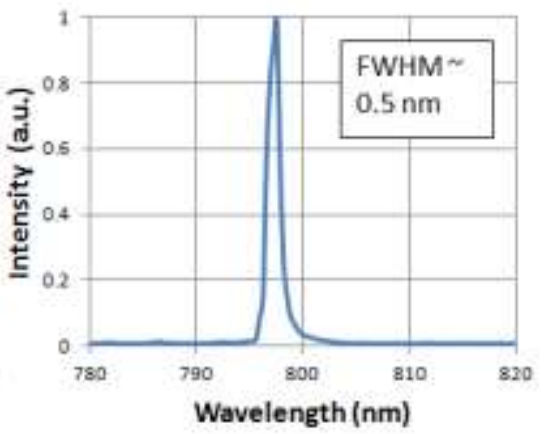

(c)

Figure 3 Measured parameters of the laser at a curved mirror separation of $8.57 \mathrm{~cm}$. Here, the laser operated in pure cw regime. (a) Oscilloscope trace of the laser output shows constant laser output power. (b) Fast Fourier transform of the measured laser output shows no tendency towards q-switching or other types of pulsed operation. Inset figure shows the lateral mode of the laser, which has $\mathrm{TEM}_{00}$ intensity distribution. (c) Measured optical spectrum was centered around $797 \mathrm{~nm}$ and had a spectral width (FWHM) of about $0.5 \mathrm{~nm}$.

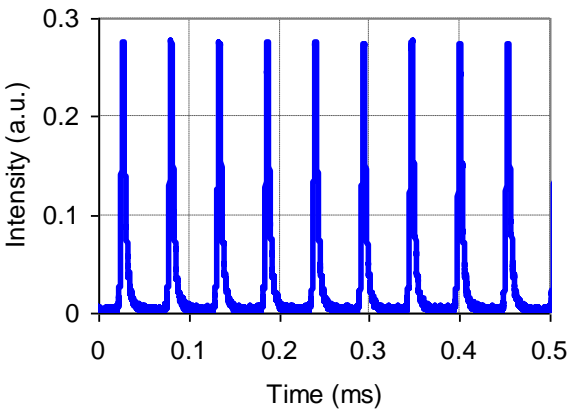

(a)

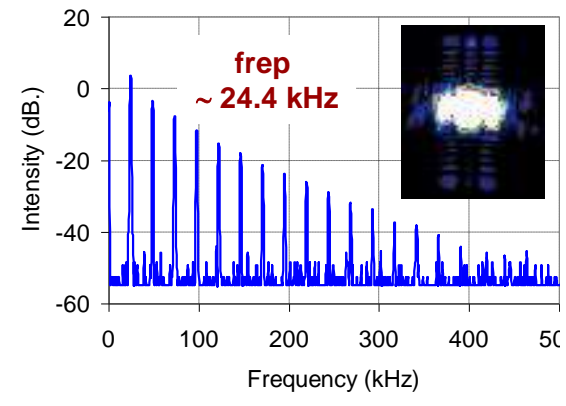

(b)

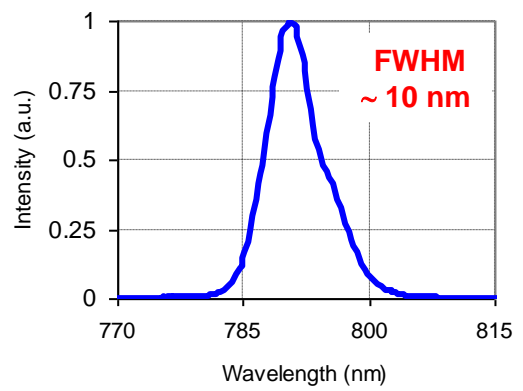

(c)

Figure 4 Measured parameters of the laser at a curved mirror separation of $8.46 \mathrm{~cm}$. The laser operated in SQS regime with an average output power of $43 \mathrm{~mW}$. (a) Oscilloscope trace of the laser output shows clean Q-switched pulses with a duration of 4.2 microseconds. (b) Fast Fourier transform of the measured laser output shows pulsing at $24.4 \mathrm{kHz}$. Inset figure shows the lateral mode of the laser. (c) Measured optical spectrum was centered near $794 \mathrm{~nm}$ and had a spectral width (FWHM) of about $10 \mathrm{~nm}$.

Table 1 Laser output parameters of the self Q-switched Cr:LiCAF laser at different curved mirror separations taken with a $0.75 \%$ transmitting output coupler.

\begin{tabular}{|c|c|c|c|}
\hline $\begin{array}{c}\text { Curved Mirror } \\
\text { Separation }(\mathrm{cm})\end{array}$ & $\begin{array}{c}\text { Spectral } \\
\text { Width }(\mathrm{nm})\end{array}$ & $\begin{array}{c}\text { Repetition } \\
\text { Rate }(\mathrm{kHz})\end{array}$ & $\begin{array}{c}\text { Output } \\
\text { Power }(\mathrm{mW})\end{array}$ \\
\hline 8.63 & 2 & 22.5 & 42 \\
\hline 8.5 & 6 & 10.35 & 37 \\
\hline 8.46 & 10 & 24.4 & 43 \\
\hline
\end{tabular}

Table 1 shows the laser output parameters of the self Q-switched Cr:LiCAF laser at different curved mirror separations. The output coupler transmission was $0.75 \%$. As can be seen, SQS operation parameters vary at different curved mirror separations. For example, a lower repetition frequency was observed at the mirror separation of $8.5 \mathrm{~cm}$ Moreover, the spectral width during SQS operation varied between $2 \mathrm{~nm}$ and $10 \mathrm{~nm}$. It should be noted here that, once the correct curved mirror separation was reached, the SQS operation was in general quite stable, meaning that the laser stayed in the SQS regime and did not make a transition from SQS to regular cw operation. 
Table 2 Laser output parameters of the self Q-switched Cr:LiCAF laser for different output couplers.

\begin{tabular}{|c|c|c|c|}
\hline $\begin{array}{c}\text { Output } \\
\text { Coupling }\end{array}$ & $\begin{array}{c}\text { Curved Mirror } \\
\text { Separation }(\mathrm{cm})\end{array}$ & $\begin{array}{c}\text { Spectral } \\
\text { Width }(\mathrm{nm})\end{array}$ & $\begin{array}{c}\text { Repetition } \\
\text { Rate }(\mathrm{kHz})\end{array}$ \\
\hline 0.25 & 8.4 & 12.5 & 19.7 \\
\hline 0.75 & 8.67 & 10 & 20.2 \\
\hline 3 & 8.54 & 2.5 & 31 \\
\hline
\end{tabular}

We note that the SQS operation could also be attained with output couplers having different transmission. As an example, Table 2 lists the laser output parameters of the self Q-switched Cr:LiCAF laser with $0.25 \%, 0.75 \%$, and 3\% output couplers. The data were recorded at the curved mirror separations that gave the maximum spectral broadening. Comparing these results, we can qualitatively note that the output spectral broadening gets smaller with decreasing output coupling as can be seen from Fig 5. It should also be noted that the center wavelength of the spectrum was different for different output couplers. In contrast, the repetition rate of the self Q-switched Cr:LiCAF laser decreases with decreasing output coupling. Hence, different output coupling levels may be needed to generate self Q-switched pulses with a desired set of temporal and spectral parameters.

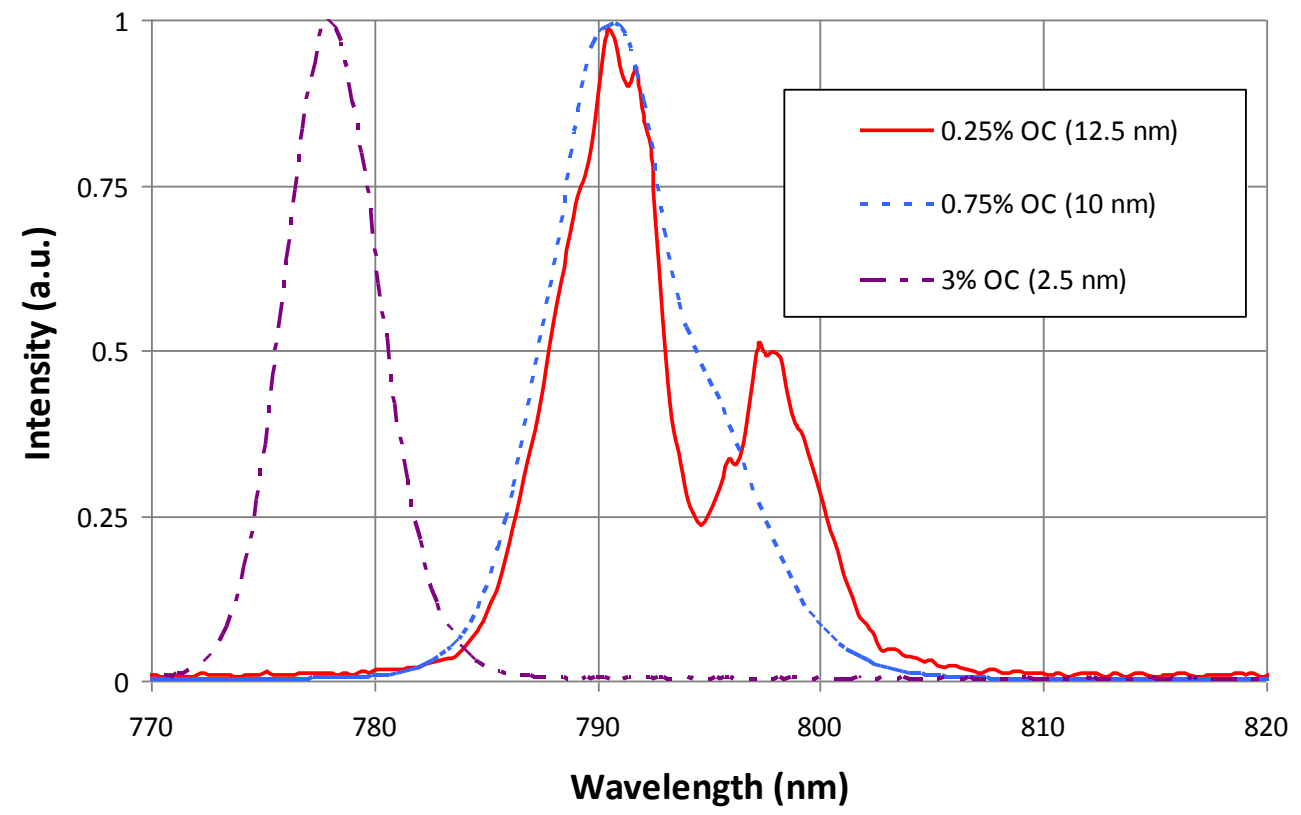

Figure 5. Spectral broadening of the self Q-switched Cr:LiCAF laser observed with different output couplers.

\section{CONCLUSIONS}

In summary, to the best of our knowledge, this study reports the SQS operation of a Cr:LiCAF laser for the first time. Different from most of the earlier studies, initiation of the SQS effect in this study did not require any misalignment of the cavity optics or cooling of the laser crystal. Furthermore, SQS operation was demonstrated with a low-cost architecture involving a low-power single-mode pump diode. Pulsing was readily obtained in a repeatable manner after fine adjustment of the curved mirror separation within the cavity stability range. Moreover, the pulsing mechanism could be observed at different output coupling levels of $0.25,0.75$, and $3 \%$. Spectral broadening up to $12.5 \mathrm{~nm}$ was observed 
with the $0.25 \%$ output coupler. We expect that the experimental data presented in this study will help in better understanding of the mechanisms responsible for the SQS effect in solid-state lasers.

We acknowledge partial support from TUBITAK (The Scientific and Technical Research Council of Turkey, 121T220), European Union Marie Curie Career Integration Grant (PCIG11-GA-2012-321787), Alexander von Humboldt-Foundation, and Center for Applied Photonics of Konstanz University.

\section{REFERENCES}

[1] A. Sennaroglu, "Broadly tunable Cr4+ doped solid-state lasers in the near infrared and visible," Progress in Quantum Electronics, vol. 26, pp. 287-352, 2002.

[2] A. V. Podlipensky, V. G. Shcherbitsky, N. V. Kuleshov, V. P. Mikhailov, V. I. Levchenko, and V. N. Yakimovich, "Cr2+:ZnSe and Co2+:ZnSe saturable-absorber Q switches for 1.54- $\mu \mathrm{m}$ Er:glass lasers," Optics Letters, vol. 24, pp. 960-962, 1999.

[3] H. Cankaya, U. Demirbas, A. K. Erdamar, and A. Sennaroglu, "Absorption saturation analysis of Cr2+: ZnSe and Fe2+: ZnSe," Journal of the Optical Society of America B-Optical Physics, vol. 25, pp. 794-800, May 2008.

[4] I. Freund, "SELF-Q-SWITCHING IN RUBY LASERS," Applied Physics Letters, vol. 12, pp. 388-\&, 1968.

[5] R. J. Collins, L. O. Braun, and D. R. Dean, "A NEW METHOD OF GIANT PULSING RUBY LASERS," Applied Physics Letters, vol. 12, pp. 392-\&, 1968.

[6] M. Birnbaum and C. L. Fincher, "THE RUBY LASER: PUMPED BY A PULSED ARGON ION LASER " Applied Physics Letters, vol. 12, pp. 225-227, 1968.

[7] A. Szabo and L. E. Erickson, "SELF-Q-SWITCHING OF RUBY LASERS AT 77 DEGREES K," IEEE Journal of Quantum Electronics, vol. QE 4, pp. 692-\&, 1968.

[8] M. Birnbaum and C. L. Fincher, "SELF-Q-SWITCHED ND3+ - YAG AND RUBY LASERS," Proceedings of the IEEE, vol. 57, pp. 804-\&, 1969.

[9] H. Samelson, A. Lempicki, and V. Brophy, "SELF-Q-SWITCHING OF ND3+ SEOCL2 LIQUID LASER," Journal of Applied Physics, vol. 39, pp. 4029-\&, 1968.

[10] Z. Zalevsky, Y. Kapellner, I. Eyal, and N. Cohen, "Self Q-switching effect in a Nd : YVO4/KTP lasing unit," Optical Engineering, vol. 45, Jul 2006.

[11] A. Szabo, "REPETITIVE SELF-Q-SWITCHING IN A CONTINUOUSLY PUMPED RUBY-LASER," Journal of Applied Physics, vol. 49, pp. 533-538, 1978.

[12] B. C. Weber and A. Hirth, "Efficient single-pulse emission with submicrosecond duration from a Cr:LiSAF laser," Optics Communications, vol. 128, pp. 158-165, Jul 11996.

[13] B. C. Weber and A. Hirth, "Presentation of a new and simple technique of Q-switching with a LiSrAlf(6): Cr3+ oscillator," Optics Communications, vol. 149, pp. 301-306, Apr 151998.

[14] R. S. Conroy, T. Lake, G. J. Friel, A. J. Kemp, and B. D. Sinclair, "Self-Q-switched Nd:YVO $\mathrm{Y}_{4}$ microchip lasers," Optics Letters, vol. 23, pp. 457-459, March 15, 19981998.

[15] A. V. Kir'yanov, N. N. Il'ichev, and Y. O. Barmenkov, "Excited-state absorption as a source of nonlinear thermo-induced lensing and self-Q-switching in an all-fiber Erbium laser," Laser Physics Letters, vol. 1, pp. 194-198, 2004.

[16] S. Wolff, A. Rodionov, V. E. Sherstobitov, C. Doering, and H. Fouckhardt, "Self-pulsation in broad area lasers with transverse-mode selective feedback," Optics Communication, vol. 265, pp. 642-648, 2006.

[17] B. N. Upadhyaya, A. Kuruvilla, U. Chakravarty, M. R. Shenoy, K. Thyagarajan, and S. M. Oak, "Effect of laser linewidth and fiber length on self-pulsing dynamics and output stabilization of single-mode Yb-doped double-clad fiber laser," Applied Optics, vol. 49, pp. 2316-2325, 2010.

[18] Y. Tang and J. Xu, "Effects of excited-state absorption on self-pulsing in Tm3+-doped fiber lasers," Journal of Optical Society of America B, vol. 27, pp. 179-1862010.

[19] N. Passilly, E. Haouas, V. Ménard, R. Moncorgé, and K. Aı"t-Ameur, "Population lensing effect in Cr:LiSAF probed by Z-scan technique," Optics Communications, vol. 260, pp. 703-707, 2006.

[20] N. Passilly, M. Fromager, and K. Ait-Ameur, "Improvement of the self-Q-switching behavior of a $\mathrm{Cr}$ : LiSrAlF6 laser by use of binary diffractive optics," Applied Optics, vol. 43, pp. 5047-5059, Sep 102004. 
[21] N. Passilly, M. Fromager, K. Ait-Ameur, R. Moncorge, J. L. Doualan, A. Hirth, and G. Quarles, "Experimental and theoretical investigation of a rapidly varying nonlinear lensing effect observed in a $\mathrm{Cr} 3+$ : LiSAF laser," Journal of the Optical Society of America B-Optical Physics, vol. 21, pp. 531-538, Mar 2004.

[22] M. Fromager and K. A. Ameur, "Modeling of the self-Q-switching behavior of lasers based on chromium doped active material," Optics Communications, vol. 191, pp. 305-314, May 82001.

[23] T. Godin, R. Moncorgé, J.-L. Doualan, M. Fromager, K. Ait-Ameur, R. A. Cruz, and T. Catunda, "Optically pump-induced athermal and nonresonant refractive index changes in the reference Cr-doped laser materials: Cr:GSGG and ruby," Journal of Optical Society of America B, vol. 29, pp. 1055-1064, 2012.

[24] U. Demirbas, R. Uecker, D. Klimm, and J. Wang, "A low-cost, broadly-tunable (375-433 nm \& 746-887 nm) Cr:LiCAF laser pumped by one single-spatial-mode diode " Applied Optics, vol. 51, pp. 8440-8448, 2012.

[25] J. G. Fujimoto, C. Pitris, S. A. Boppart, and M. E. Brezinski, "Optical Coherence Tomography: An Emerging Technology for Biomedical Imaging and Optical Biopsy," Neoplasia, vol. 2, pp. 9-25, 2000. 